\title{
Statistical Iterative Reconstruction \\ Algorithm Based on a \\ Continuous-to-Continuous Model \\ Formulated for Spiral Cone-Beam CT
}

\author{
Robert Cierniak $^{(\otimes)}{ }^{(\mathbb{D})}$ and Piotr Pluta \\ Institute of Computational Intelligence, Czestochowa University of Technology, \\ Armii Krajowej 36, 42-200 Czestochowa, Poland \\ robert.cierniak@pcz.pl
}

\begin{abstract}
This paper is closely related to the originally formulated 3D statistical model-based iterative reconstruction algorithm for spiral cone-beam x-ray tomography. The concept proposed here is based on a continuous-to-continuous data model, and the reconstruction problem is formulated as a shift invariant system. This algorithm significantly improves the quality of the subsequently reconstructed images, so allowing a reduction in the x-ray dose absorbed by a patient. This form of reconstruction problem permits a reduction in the computational complexity in comparison with other model-based iterative approaches. Computer simulations have shown that the reconstruction method presented here outperforms standard FDK methods with regard to the image quality obtained and can be competitive in terms of time of calculation.
\end{abstract}

Keywords: Iterative reconstruction algorithm $\cdot$ Computed tomography $\cdot$ Statistical method

\section{Introduction}

Recently, the most significant problem in medical CT has been the development of image reconstruction methods which would enable the reduction of the impact of measurement noise on the quality of tomography images and thus decrease the dose of X-ray radiation absorbed by patients during examinations. Some of the most interesting research directions in this area are statistical reconstruction methods, especially those belonging to the MBIR (Model-Based Iterative Reconstruction) approach [1-3], where a probabilistic model of the measurement signals is taken into account. The objective in those solutions was devised

\footnotetext{
Supported by The National Centre for Research and Development in Poland (Research Project POIR.01.01.01-00-0463/17.).

(C) Springer Nature Switzerland AG 2020

V. V. Krzhizhanovskaya et al. (Eds.): ICCS 2020, LNCS 12139, pp. 613-620, 2020.

https://doi.org/10.1007/978-3-030-50420-5_46
} 
according to a discrete-to-discrete (D-D) data model. Unfortunately, those methods have some very serious drawbacks from the theoretical and practical point of view: for instance, if the image resolution is set to be $\mathrm{I} \times \mathrm{I}$ pixels, the calculation complexity of the problem is proportional to $I^{4}$, the statistical reconstruction procedure based on this methodology necessitates simultaneous calculations for all the voxels in the range of the reconstructed 3D image, the size of the forward model matrix $\mathbf{A}$ is huge, and this makes it often necessary to calculate them in every iteration of the reconstruction algorithm. In this case, the reconstruction problem is extremely ill-conditioned, and it is necessary to introduce an a priori term (often referred to in the literature as a regularization term) into the objective, and this leads to the use of the MAP model. The problems connected with the use of a methodology based on the D-D data model can be reduced by using a strategy of reconstructed image processing based on a continuous-tocontinuous (C-C) data model. In previous papers we have shown how to formulate reconstruction problems consistent with the ML methodology for parallel scanner geometry [4], and finally for the spiral cone-beam scanner [5,6]. In this paper, we show how to interpret our original statistical reconstruction method as an approach belonging to the $\mathrm{C}-\mathrm{C}$ mode. We applied very popular and convenient reconstruction strategy, which resemles the FDK-type algorithms, and we present a conception of the direct use of spiral cone-beam projections to a statistical reconstruction algorithm based on the $\mathrm{C}-\mathrm{C}$ data model.

\section{Statistical Reconstruction Algorithm}

Our reconstruction method is based on the well-known maximum-likelihood (ML) estimation [8]. In most cases, the objective in those solutions is devised according to a discrete-to-discrete (D-D) data model. We propose here an optimization formula which is consistent with the $\mathrm{C}-\mathrm{C}$ data model, in the following form:

$$
\mu_{\min }=\arg \min _{\mu}\left(\int_{x} \int_{y}\left(\int_{\bar{x}} \int_{\bar{y}} \mu(\bar{x}, \bar{y}) \cdot h_{\Delta x, \Delta y} d \bar{x} d \bar{y}-\tilde{\mu}(x, y)\right)^{2} d x d y\right),
$$

where $\tilde{\mu}(x, y)$ is an image obtained by way of a back-projection operation, obtained theoretically in the following way:

$$
\tilde{\mu}(x, y) \approx \int_{0}^{2 \pi} \int_{-\beta_{\max }}^{\beta_{\max }} p^{h}\left(\beta, \alpha^{h}, z_{k}\right) \frac{R_{f d}}{\sqrt{R_{f d}^{2}+z_{k}^{2}}} \operatorname{int}_{L}(\Delta \beta) d \beta d \alpha,
$$

wherein $p^{h}\left(\beta, \alpha^{h}, z_{k}\right)$ are measurements carried out using a spiral cone-beam scanner, $R_{f d}$ is the SDD (Source-to-Detector Distance), and the coefficients $h_{\Delta i, \Delta j}$ can be precalculated according to the following relation: 


$$
h_{\Delta x, \Delta y}=\int_{0}^{2 \pi} \operatorname{int}(\Delta x \cos \alpha+\Delta y \sin \alpha) d \alpha,
$$

and $\operatorname{int}(\Delta s)$ is a linear interpolation function.

The necessary measurements are performed in a standard helical cone-beam scanner. The mesurement system consists of an x-ray tube and a rigidly coupled screen with a multi-row matrix of detectors. This assembly rotates around the $z$-axis (the principal axis of the system) and at the same time, the patient table moves into the gantry. Therefore, the moving projection system traces a spiral path around the $z$-axis. Each ray emitted by the tube at a particular angle of rotation and reaching any of the radiation detectors can be identified by $\left(\beta, \alpha^{h}, \dot{z}\right)$, as follows: $\beta$ - the angle between a particular ray in the beam and the axis of symmetry of the moving projection system; $\alpha^{h}-$ the angle at which the projection is made, i.e. the angle between the axis of symmetry of the rotated projection system and the $y$-axis; $\dot{z}$ - the $z$-coordinate relative to the current position of the moving projection system.

In a real spiral cone-beam scanner, the reconstruction algorithm can only make use of projections obtained at certain angles and measured only at particular points on the screen. Let us assume that the beam of x-rays reaches the individual detector rows $k=1,2, \ldots, \mathrm{K}$, where $\mathrm{K}$ is a number of detectors in each row of the array. In every row, selected rays strike the detectors, each of which is described by the index $\eta=-(\mathrm{H}-1) / 2, \ldots, 0, \ldots,(\mathrm{H}-1) / 2$, where $\mathrm{H}$ is a number of detectors in each channel of the array. Detectors are placed on the screen separated by a distance $\Delta_{z}$ in each row, and by an angular distance $\Delta_{\eta}$ in each channel. Of course, only a limited number of mesurements are performed, each of which is described by the index $\theta=0, \ldots, \Theta-1$, where $\Theta-1$ is the total number of projections made during the examination. Every projection is carried out after rotation by $\Delta_{\theta}$. We can sum up above conditions by saying that the reconstruction algorithm has available to it the projection values $p^{h}\left(\beta_{\eta}, \alpha_{\theta}^{h}, \dot{z}_{k}\right)$, in the ranges: $\eta=-(\mathrm{H}-1) / 2, \ldots, 0, \ldots,(\mathrm{H}-1) / 2$; $\theta=0, \ldots, \Theta-1 ; k=1,2, \ldots, \mathrm{K}$.

According to the originally formulated by us iterative approach to the reconstruction problem, decribed by Eqs. (1)-(3), it is possible to present a practical model-based statistical method of image reconstruction, as follows:

$$
\mu_{\min }=\arg \min _{\mu}\left(\sum_{i=1}^{\mathrm{I}} \sum_{j=1}^{\mathrm{J}}\left(\sum_{\bar{i}} \sum_{\bar{j}} \mu^{*}\left(x_{\bar{i}}, y_{\bar{j}}\right) \cdot h_{\Delta i, \Delta j}-\tilde{\mu}\left(x_{i}, y_{j}\right)\right)^{2}\right)
$$

and $\tilde{\mu}(i, j)$ is an image obtained by way of a back-projection operation, in the following way:

$$
\tilde{\mu}\left(x_{i}, y_{j}\right)=\Delta_{\alpha^{h}} \sum_{\theta} \dot{p}^{h}\left(\beta_{i j}, \alpha_{\theta}^{h}, \dot{z}_{i j}\right)
$$


It is necessary to use an interpolation to evaluate projections at points $\beta_{i j}$ based on the measured projections $p^{h}\left(\beta_{\eta}, \alpha_{\theta}^{h}, \dot{z}_{k}\right)$. We can obtain an approximations of these projections as follows:

$$
\begin{aligned}
& \dot{p}^{h}\left(\beta_{i j}, \alpha_{\theta}^{h}, \dot{z}_{i j}\right)= \\
& \sum_{k} \sum_{\eta} p^{h}\left(\beta_{\eta}, \alpha_{\theta}^{h}, \dot{z}_{k}\right) \frac{R_{f d}}{\sqrt{R_{f d}^{2}+z_{k}^{2}}} \cdot \operatorname{int}_{\beta}\left(\beta_{i j}-\eta \Delta_{\beta}\right) \text { int }_{z}\left(z_{i j}-k \Delta_{z}\right),
\end{aligned}
$$

where $\operatorname{int}_{\beta}(\Delta \beta)$ and $\operatorname{int}_{z}(\Delta z)$ are the interpolation functions, i.e. in the simplest case, linear interpolations:

$$
\operatorname{int}_{\beta}(\beta)=\left\{\begin{array}{ll}
\frac{1}{\Delta_{\beta}}\left(1-\frac{|\beta|}{\Delta_{\beta}}\right) & \text { for }|\beta| \leq \Delta_{\beta} \\
0 & \text { for }|\beta| \geq \Delta_{\beta}
\end{array},\right.
$$

and

$$
\operatorname{int}_{z}(z)= \begin{cases}\frac{1}{\Delta_{z}}\left(1-\frac{|z|}{\Delta_{z}}\right) & \text { for }|z| \leq \Delta_{z} \\ 0 & \text { for }|z| \geq \Delta_{z}\end{cases}
$$

The coefficients $h_{\Delta i, \Delta j}$ are determined according to the following formula:

$$
h_{\Delta i, \Delta j}=\frac{1}{\Delta s^{2}} \Delta_{\alpha} \sum_{\psi=0}^{\Psi-1} \operatorname{int}\left(\Delta i \cos \psi \Delta_{\alpha}+\Delta j \sin \psi \Delta_{\alpha}\right) \text {, }
$$

wherein $\operatorname{int}(\Delta s)$ is an interpolation function used in the back-projection operation, and $\Delta_{s}=R_{f} * \tan \Delta_{\beta}$.

The presence of a shift-invariant system in the optimization problem (4) implies that this system is much better conditioned than the least squares problems present in the referential approach [9]. The conception presented above is a full 3D iterative reconstruction algorithm for spiral cone-beam scanner geometry. This algorithm is based on the one of the principal reconstruction methods devised for the cone-beam spiral scanner, i.e. the generalized FDK algorithm. The statistical reconstruction method proposed by us consists of two steps, namely: a back-projection operation described by relations (5)-(8) and an iterative reconstruction procedure according to formula (4). Figure 1 depicts this algorithm after discretization and implementation of FFT which significantly accelerates the calculations (the iterative reconstruction procedure is patented in the United States [10]).

\section{Experimental Results}

In our experiments, we have used projections obtained from a Somatom Definition $\mathrm{AS}+$ (helical mode) scanner with the following parameters: reference tube potential $120 \mathrm{kVp}$ and quality reference effective $200 \mathrm{mAs}, R_{f d}=1085.6 \mathrm{~mm}$, 


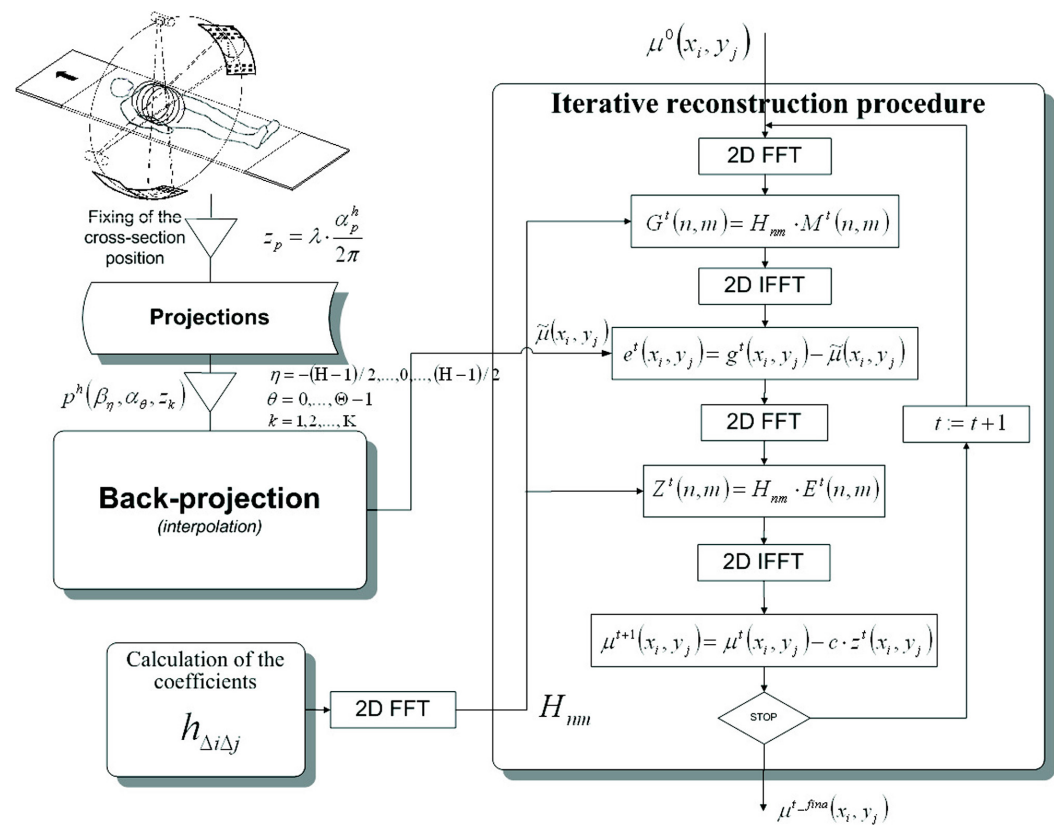

Fig. 1. Statistical reconstruction algorithm for spiral cone-beam scanner.

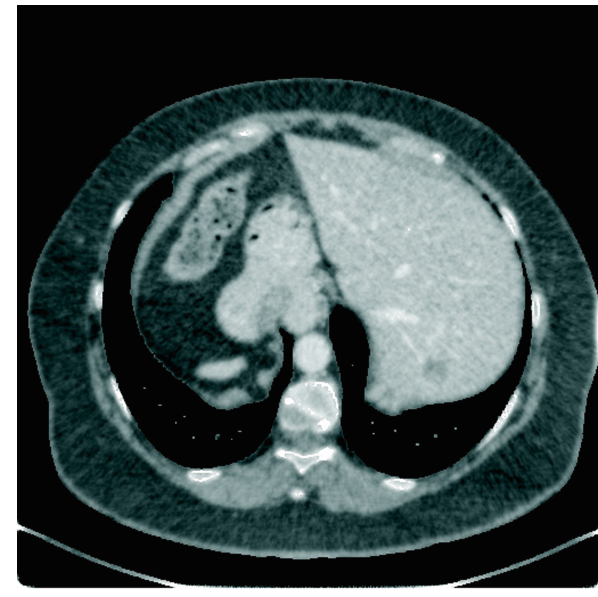

(a)

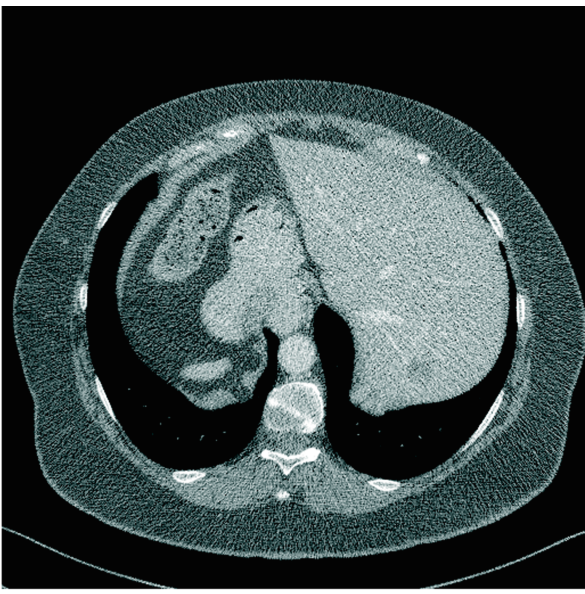

(b)

Fig. 2. Reconstructed images obtained at 50\% x-ray dose reduction, using: (a) the statistical approach presented in this paper obtained after 10000 iterations (b) the standard FDK algorithm. 


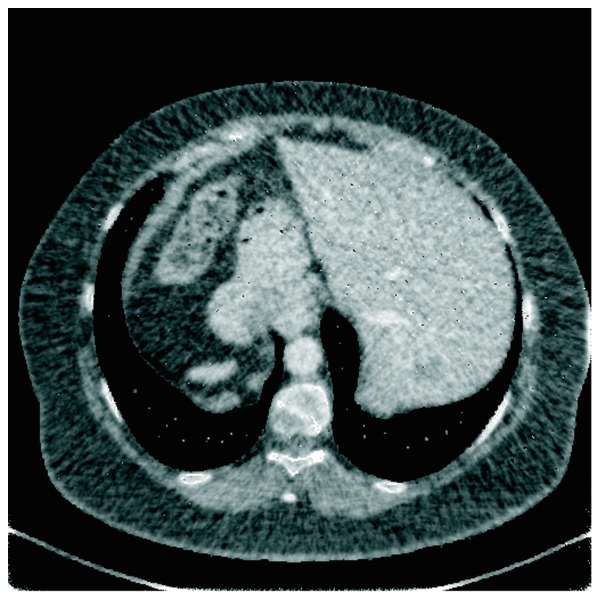

(a)

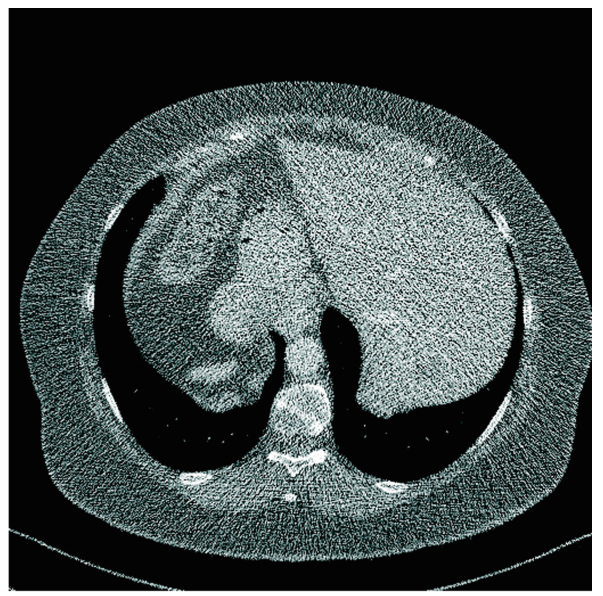

(b)

Fig. 3. Reconstructed images obtained at $87 \%$ x-ray dose reduction, using: (a) the statistical approach presented in this paper obtained after 10000 iterations (b) the standard FDK algorithm.

$\mathrm{R}_{f}=595 \mathrm{~mm}$, number of views per rotation $\Psi=1152$, number of pixels in detector panel 736 , detector dimensions were $1.09 \mathrm{~mm} \times 1.28 \mathrm{~mm}$. However, these projections were performed using two flying focal spots but only measurements carried out which coincided with the detector's center were used. This means that only every second measurement (theoretically, a $50 \%$ reduction of the dose) was useful for the reconstruction algorithm proposed by us. During the experiments, the size of the processed image was fixed at $512 \times 512$ pixels. A discrete representation of the matrix $h_{\Delta x, \Delta y}$ was established before the reconstruction process was started, and these coefficients were fixed (transformed into the frequency domain) for the whole iterative reconstruction procedure. The image obtained after the back-projection operation was then subjected to a process of reconstruction (optimization) using an iterative procedure. A specially prepared result of an FBP reconstruction algorithm was chosen as the starting point of this procedure. It is worth noting that our reconstruction procedure was performed without any regularization regarding the objective function from (1). The iterative reconstruction procedure was implemented for a computer with 10 cores, i.e. with an Intel i9-7900X BOX/3800MHz processor (the iterative reconstruction procedure was implemented at assembler level), and using a GPU type nVidia Titan V. According to an assessment of the quality of the obtained images by a radiologist, 8000 iterations are enough to provide an acceptable image. The same results were achieved for both hardware implementations after $7.44 \mathrm{~s}$ and $7.73 \mathrm{~s}$, for the CPU and GPU implementations, respectively. One can compare the results obtained by assessing the views of the reconstructed images in Figs. $2 \mathrm{a}$ and $3 \mathrm{a}$, where the statistical approach presented in this paper was used (image 
obtained after 10000 iterations), and in Figs. 2b and 3b, where the standard FDK algorithm was applied (with linear interpolation function and Shepp-Logan kernel). Figures 2 and 3 show reconstructed images obtained at 50\% x-ray dose reduction, and at $87 \% \mathrm{x}$-ray dose reduction, respectively.

\section{Conclusion}

A statistical iterative reconstruction algorithm which can be used in practice for helical cone-beam scanners has been shown above. We have conducted computer simulations, which proved that our reconstruction method is very fast, above all thanks to the use of FFT algorithms and efficient programming techniques, and it gives satisfactory results regarding the quality of the obtained images at a significantly reduced dose of $\mathrm{x}$-rays absorbed by the patient. If the image resolution is assumed to be $\mathrm{I} \times \mathrm{I}$ pixels, the complexity of the approach implemented here is proportional to $I^{2} \log _{2} I$, and with the referential approach it is of the level of $I^{4} \times$ number_of_cross - sections. One can note that the iterative reconstruction procedure was performed without introducing any additional regularization term, using only an early stopping regularization strategy. It should be underlined that the price of the hardware used is relatively low (about 5000 USD in both cases) compared with the cost of the equipment necessary in the case of the referential solution.

\section{References}

1. Zhou, Y., Thibault, J.-B., Bouman, C.A., Hsieh, J., Sauer, K.D.: Fast model-based $\mathrm{x}$-ray CT reconstruction using spatially non-homogeneous ICD optimization. IEEE Tran. Image Proc. 20, 161-175 (2011)

2. Ding, Q., Long, Y., Zhang, X., Fessler, J.A.: Modeling mixed Poisson-Gaussian noise in statistical image reconstruction for x-ray CT. In: Proceedings of the 4th International Conference on Image Formation in X-Ray Computed Tomography, Bamberg, Germany, pp. 399-402 (2016)

3. Geyer, L.L., et al.: State of the art: iterative CT reconstruction techniques. Radiology 276, 339-357 (2017)

4. Cierniak, R.: An analytical iterative statistical algorithm for image reconstruction from projections. Appl. Math. Comput. Sci. 24, 7-17 (2014)

5. Cierniak, R.: Analytical statistical reconstruction algorithm with the direct use of projections performed in spiral cone-beam scanners. In: Proceedings of the 5th International Meeting on Image Formation in X-Ray Computed Tomography, pp. 293-296, Salt Lake City (2018)

6. Cierniak, R., Pluta, P., Kaźmierczak, A.: A practical statistical approach to the reconstruction problem using a single slice rebinning method. J. Artif. Intell. Soft Comput. Res. 10, 137-149 (2020)

7. Feldkamp, L.A., Davis, L.C., Kress, J.W.: Practical cone-beam algorithm. J. Opti. Soc. Am. 1(A) 9, 612-619 (1984)

8. Bouman, C.A., Sauer, K.: A unified approach to statistical tomography using coordinate descent optimization. IEEE Tran. Image Proc. 5, 480-492 (1996) 
9. Cierniak, R., Lorent, A.: Comparison of algebraic and analytical approaches to the formulation of the statistical model-based reconstruction problem for x-ray computed tomography. Comput. Med. Imaging Graph. 52, 19-27 (2016)

10. Cierniak R.: Fast iterative reconstruction method for 3D computed tomography. Patent US 9.508.164 B2 (2016) 